\title{
The M35 Metalloprotease Effector FocM35_1 Is Required for Full Virulence of Fusarium oxysporum f. sp. cubense Tropical Race 4
}

\author{
Xiaoxia Zhang ${ }^{1,+}{ }^{+}$Huoqing Huang ${ }^{2,+}$, Bangting $\mathrm{Wu}^{2,+}{ }^{2}$ Jianghui $\mathrm{Xie}^{3}{ }^{3}$, Altus Viljoen ${ }^{4}$, Wei Wang ${ }^{3}$, \\ Diane Mostert ${ }^{4}\left(\mathbb{D}\right.$, Yanling Xie ${ }^{2}$, Gang Fu ${ }^{5}$, Dandan Xiang ${ }^{2}\left(\mathbb{D}\right.$, Shuxia Lyu ${ }^{1, *}$, Siwen Liu ${ }^{2, *(1)}$ and Chunyu $\mathrm{Li}^{2}$
}

check for updates

Citation: Zhang, X.; Huang, H.; Wu, B.; Xie, J.; Viljoen, A.; Wang, W.;

Mostert, D.; Xie, Y.; Fu, G.; Xiang, D.; et al. The M35 Metalloprotease Effector FocM35_1 Is Required for Full Virulence of Fusarium oxysporum f. sp. cubense Tropical Race 4. Pathogens 2021, 10, 670. https:// doi.org/10.3390/pathogens10060670

Academic Editor: Flora Pensec

Received: 22 April 2021

Accepted: 26 May 2021

Published: 29 May 2021

Publisher's Note: MDPI stays neutral with regard to jurisdictional claims in published maps and institutional affiliations.

Copyright: (C) 2021 by the authors Licensee MDPI, Basel, Switzerland. This article is an open access article distributed under the terms and conditions of the Creative Commons Attribution (CC BY) license (https:/ / creativecommons.org/licenses/by/ $4.0 /)$.
1 College of Bioscience and Biotechnology, Shenyang Agricultural University, Shenyang 110866, China; zhangxiaoxia418@163.com

2 Key Laboratory of South Subtropical Fruit Biology and Genetic Resource Utilization, Ministry of Agriculture, Key Laboratory of Tropical and Subtropical Fruit Tree Research of Guangdong Province, Institution of Fruit Tree Research, Guangdong Academy of Agricultural Sciences, Guangzhou 510640, China;

hqhuang07@163.com (H.H.); bangtingwu@163.com (B.W.); xieyanling19@webmail.hzau.edu.cn (Y.X.); xiangdandan@gdaas.cn (D.X.); lichunyu@gdaas.cn (C.L.)

3 Key Laboratory of Biology and Genetic Resources of Tropical Crops, Ministry of Agriculture, Institute of Tropical Bioscience and Biotechnology, Chinese Academy of Tropical Agricultural Sciences, Haikou 571101, China; xiejianghui@itbb.org.cn (J.X.); wangwei@itbb.org.cn (W.W.)

4 Department of Plant Pathology, University of Stellenbosch, Matieland 7602, South Africa; altus@sun.ac.za (A.V.); diane@sun.ac.za (D.M.)

5 Institute of Plant Protection, Guangxi Academy of Agricultural Sciences, Guangxi Key Laboratory of Biology for Crop Diseases and Insect Pests, Nanning 530007, China; fug110@gxaas.net

* Correspondence: lushuxia@syau.edu.cn (S.L.); liusiwen@gdaas.cn (S.L.); Tel.: +86-1332-246-2868 (Shuxia Lyu)

+ These authors contributed equally to this work.

Abstract: Fusarium oxysporum f. sp. cubense tropical race 4 (Foc TR4) causes Fusarium wilt of banana, the most devastating disease on a banana plant. The genome of Foc TR4 encodes many candidate effector proteins. However, little is known about the functions of these effector proteins on their contributions to disease development and Foc TR4 virulence. Here, we discovered a secreted metalloprotease, FocM35_1, which is an essential virulence effector of Foc TR4. FocM35_1 was highly upregulated during the early stages of Foc TR4 infection progress in bananas. The FocM35_1 knockout mutant compromised the virulence of Foc TR4. FocM35_1 could interact with the banana chitinase MaChiA, and it decreased banana chitinase activity. FocM35_1 induced cell death in Nicotiana benthamiana while suppressing the INF1-induced hypersensitive response (HR), and its predicted enzymatic site was required for lesion formation and the suppression to INF1-induced HR on N. benthamiana leaves. Importantly, treatment of banana leaves with recombinant FocM35_1 accelerates Foc TR4 infection. Collectively, our study provides evidence that metalloprotease effector FocM35 seems to contribute to pathogen virulence by inhibiting the host immunity.

Keywords: Fusarium wilt; banana; virulence; effector; metalloprotease

\section{Introduction}

Banana (Musa spp.) is one of the most important fruits and a staple diet for millions of people in the world [1]). The plant pathogen Fusarium oxysporum f. sp. cubense (Foc) causes Fusarium wilt on bananas, resulting in substantial yield losses. Four races of Foc have been classified based on different host banana types: race1, which is responsible for the Gros Michel epidemics, also attacks some AAB or ABB bananas; race 2 is pathogenic to cooking bananas such as 'Bluggoe' ABB; race 3 is no longer considered as Foc race since it only affects Heliconia species [2]; Foc race 4 is pathogenic to race 1- and race 2-susceptible cultivars as well as the Cavendish cultivars (AAA), which are grown and consumed worldwide nowadays. Foc race 4 isolates are further divided into two groups: subtropical race 4 (STR4) 
and tropical race 4 (TR4) [1]. Foc TR4 is more virulent and has spread from the Asia-Pacific region to the Middle East even to Latin America, as reported recently [1,3-5]. No efficient control measures have been developed against Foc TR4, other than the replacement of susceptible with resistant bananas or genetically modified Cavendish plants [6].

Foc TR4 is considered a hemibiotrophic pathogen [7]. Hemi-biotrophic pathogens deliver apoplastic and intracellular effector proteins to manipulate the host physiological metabolism and to induce cell death in order to facilitate pathogen colonization [8,9]. Integrating genome sequencing and bioinformatics, a large number of effector candidate genes have been predicted in Foc TR4 (data not published). However, functional analysis of predicted effector candidate genes has not been performed. So far, only a few effectors in Foc TR4 have been reported to be essential for virulence. For instance, FocSix1 and FocSix8, which are homologs of SIX (secreted in the xylem) effectors identified in F. oxysporum $\mathrm{f}$. sp. lycopersici $(F o l)$, are required for full virulence $[10,11]$. FocCP1 was demonstrated as a virulence factor that is required by Foc TR4 for penetration and full virulence [12]. However, none of these studies demonstrated the underlying molecular mechanisms involved in the host-pathogen interactions.

Metalloproteases of diverse families have been identified in various microbial pathogens with different functions in virulence contribution [13]. Among them, M35 (deuterolysin) and M36 (fungalysin) families are the two major types of metalloproteases secreted by pathogenic fungi. Fusarium verticillioides secretes Fv-cmp, a fungalysin protein with an M36 domain, to target and cleave class IV chitinases of maize at the chitin-binding domain [14]). An M36 metalloprotease UmFly of Ustilago maydis functions as a virulence factor by cleaving maize chitinase $\mathrm{ZmChiA}$ to promote fungal infection [15]. Several other plant pathogenic fungal, including Colletotrichum higginsianum, Verticillium species, and Botrytis cinerea, were also reported to cleave plant class IV Chitinase [16-18]. However, the functional roles of M35 family metalloprotease in the plant pathogenic fungi and their targets in plant remain largely unknown. M35 and M36 metalloproteases share the HEXXH motif [19], indicating M35 metalloprotease maybe also able to target class IV chitinases.

In this study, based on an earlier transcriptomics study on the infection process of banana roots by Foc TR4 (data not published), a potential secreted metalloprotease FocM35_1 was identified. It belongs to the M35 metalloprotease family that is conserved among various pathogens. It was demonstrated that FocM35_1 is a virulence factor of Foc TR4 and is able to decrease banana chitinase activity. Also, it was found that FocM35_1 was able to trigger cell death and suppressed the INF1-induced hypersensitive response in the nonhost plant Nicotiana. benthamiana. The cell death triggering effect of FocM35_1 was dependent on the predicted enzymatic site and was able to enhance the disease development during Foc TR4 infection.

\section{Results}

2.1. FocM35_1 Encodes a Metalloprotease That Is Conserved among Different Fusarium Strains

FocM35_1 was first identified from the RNA-Seq profiling of banana roots infected with Foc TR4 (data not published). Among the candidate effectors identified in II5 (specified Foc TR4 strain) genome, FocM35_1 showed continuous high expression in the transcriptome analysis. It encoded a putative protein with 351 amino acid residues and was predicted to contain an N-terminal signal peptide (SP) and an M35 domain, which has two zinc-binding histidines and catalytic residue glutamate in a HEXXH motif (Figure 1a). 
(a)

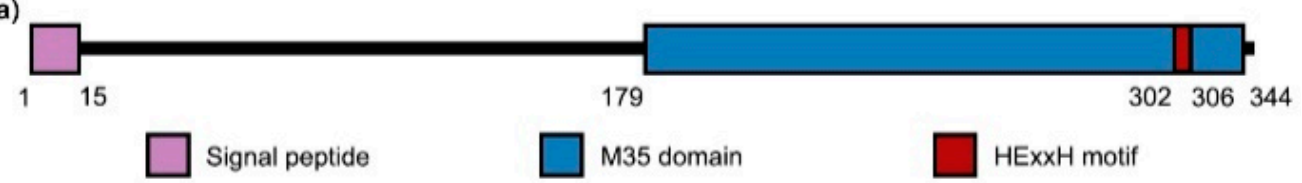

(b)

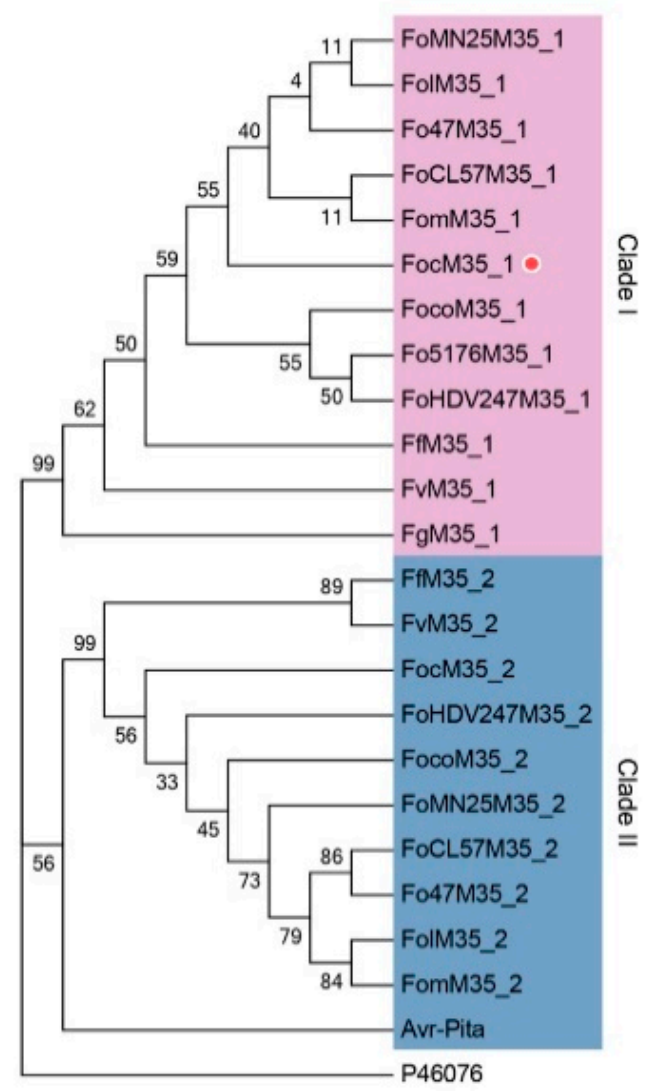

Figure 1. Sequence analysis of FocM35_1. (a) Schematic structure of the FocM35_1 protein, a M35 metalloprotease. (b) Maximum likelihood tree based on P46076 (M35 protein of Aspergillus oryzae) and Avr-Pita and their orthologous proteins from Fusarium spp. genomes. Red dot indicates FocM35_1.

A BlastP search against the National Center for Biotechnology Information (NCBI) non-redundant (nr) database identified 21 additional FocM35_1 homologs in a range of additional Fusarium species. Multiple sequence alignment of these M35 domain-containing proteins showed that they all contain a HEXXH motif, which is typical of the M35 protein family (Figure S1). Except for Fusarium graminearum and Fo5176, two orthologues of M35 protein were identified in Fusarium species that clustered into two paralogous clades (Figure 1b). Although both clade I and clade II Fusarium M35 proteins were orthologues of P46076 (M35 protein of Aspergillus oryzae) [20], members of clade I shared more sequence similarities with P46076. M35-domain containing proteins in clade II are clustered closer with Magnaporthe Avr-Pita effector (Figure 1b), suggesting they may have similar functions.

To confirm the secretory function of the predicted SP of FocM35_1, we tested the ability of the SP to direct the secretion of invertase using a yeast secretion system. Similar to Avr1b with a known functional signal peptide [21], the predicted signal peptide of FocM35_1 was able to mediate the secretion of invertase after being fused with the invertase (Figure S2), indicating FocM35_1 is indeed a secreted protein.

\subsection{FocM35_1 Contributes to Foc TR4 Virulence}

Expression levels of FocM35_1 were analyzed with qRT-PCR using samples from conidia, vegetative mycelium, and infected roots at different time points of infection. It was 
found FocM35_1 was slightly induced at $12 \mathrm{~h}$ post-inoculation (hpi) and highly induced at 48 hpi (Figure 2). Therefore, we postulated that FocM35_1 may play a role in the virulence of Foc TR4 during early infection stages on a banana.

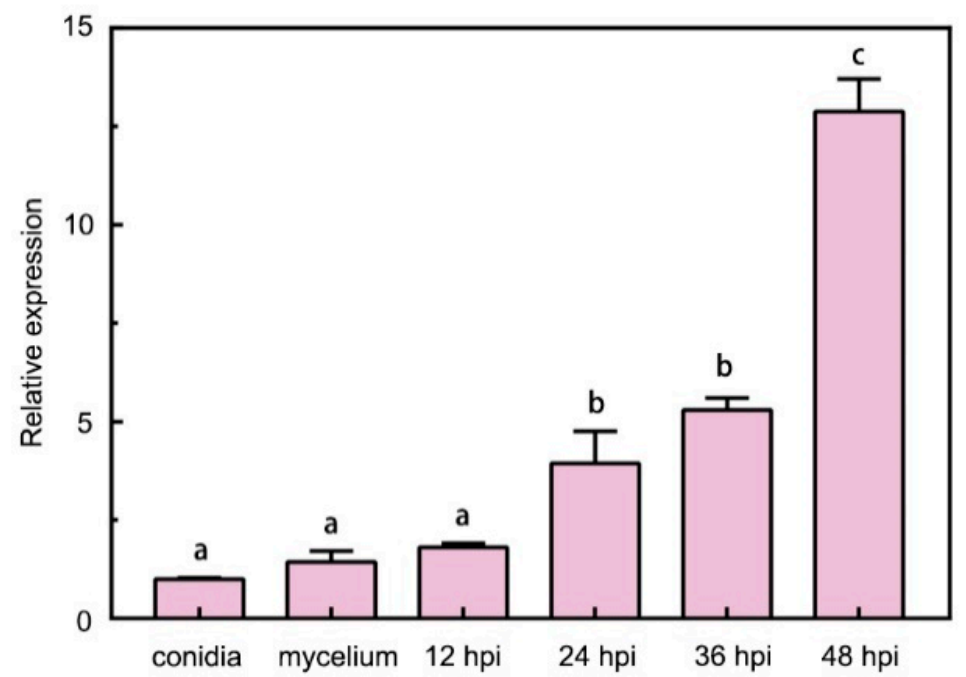

Figure 2. FocM35_1 expression at different development stages. The relative expression levels were compared to that of the conidia (set as 1). The fungal constitutive gene FocEF1 $\alpha$ was used as internal reference. Data are the means of tree independent experiments. The letters above the bars indicate statistically significant differences at $p<0.05$ (Student's $t$-test).

To determine the virulence contribution of FocM35_1 in Foc TR4, a FocM35_1-deletion mutant ( $\triangle$ FocM35_1) was obtained by replacing the FocM35_1 region with hygromycinresistance gene (HPH) in Foc TR4 reference strain II5 (Figure S3). Gene replacement mutants were preliminarily identified via PCR screening (Figure S3) using primer pairs listed in Table S1. The candidate transformant was further confirmed by Southern blot analysis using a HPH-specific probe (Figure S3). Genetic complementation of the $\Delta$ FocM35_1 mutant $(\triangle$ FocM35_1-C) was obtained by introducing into the $\Delta$ FocM35_1 strain a construct containing the FocM35_1 gene under the FocM35_1 native promoter. The complemented strains were also validated by PCR amplification (Figure S3).

The knockout mutant $\triangle$ FocM35_1 showed no differences in vegetative growth on potato dextrose agar (PDA) compared to the wild-type (WT) strain and the complementation strain $\triangle$ FocM35_1-C (Figure 3a,b). We further tested the virulence of $\Delta$ FocM35_1, $\triangle$ FocM35_1-C, and WT by root infection assays. Compared with WT and $\Delta$ FocM35_1-C, $\Delta$ FocM35_1 mutant caused significantly fewer symptoms on banana corms 30 days after inoculation of the conidia suspensions (Figure 3c). Of the banana plantlets inoculated with WT and $\triangle$ FocM35_1 mutant, $83.3 \%$ and $6.7 \%$ resulted in slight browning symptoms and no symptoms in corms, respectively. To determine whether the targeted disruption of FocM35_1 affected the fungal growth in planta, the ratio of fungal DNA to banana DNA was measured using quantitative PCR (qPCR). The biomass of $\triangle$ FocM35_1 mutant in banana corms was significantly lower than that of WT (Figure 3e). These results suggest FocM35_1 plays an essential role during the banana root infection by Foc TR4.

In order to explore the underlying mechanisms of FocM35_1 in virulence contribution, the $\Delta$ FocM35 mutant strain for its ability to penetrate into cellophane membranes and banana roots was measured. The growth of fungi on plates covered with cellophane could be used as a condition to mimic the penetration phase in vitro [22]. It was found that the ability of the $\Delta$ FocM35 mutant to penetrate cellophane was significantly reduced compared to the WT strain (Figure S4a). Consistently, the events of adhesion and penetration on a banana root surface by $\triangle$ FocM35 were also significantly reduced compared to the WT (Figure S4b). These observations suggest that the lack of FocM35_1 probably led to a decreased fungal growth within host tissues. 
(a)

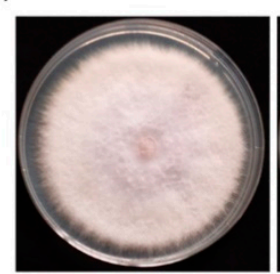

WT

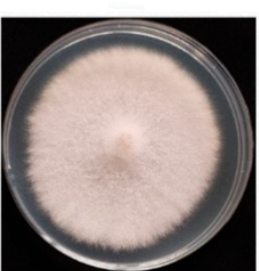

$\Delta$ FocM35_1

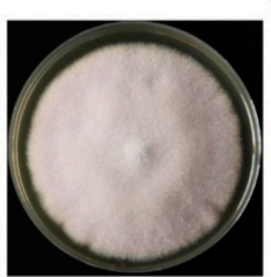

$\Delta$ FocM35_1-C

(b)

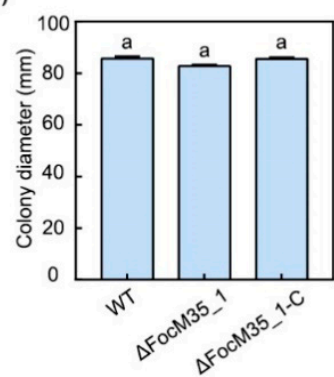

(c)

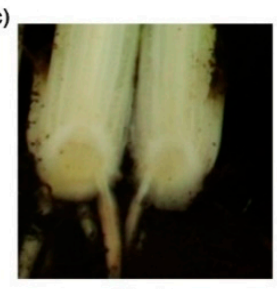

Mock

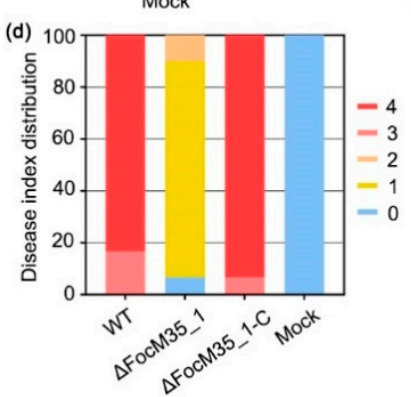

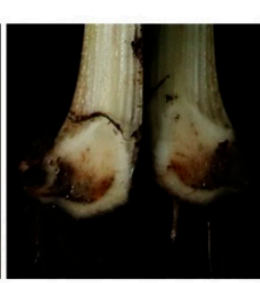

WT

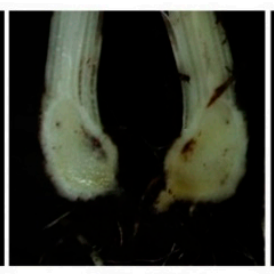

$\Delta$ FocM35_1

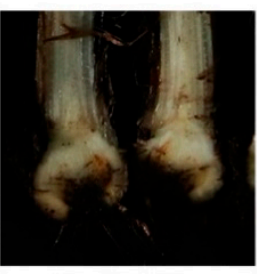

$\Delta$ FocM35_1-C

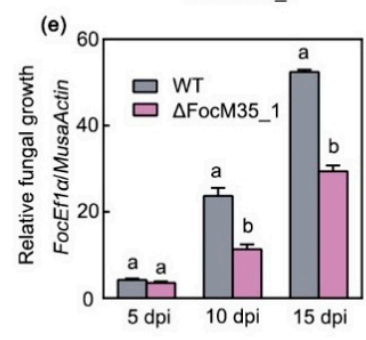

Figure 3. FocM35_1 contributes to the virulence of Foc TR4. (a) Colony morphologies of wild type (WT), $\triangle$ FocM35_1 and $\triangle$ FocM35_1-C strains cultured on potato dextrose agar (PDA) plates. Photos were taken 5 days after incubation at $28^{\circ} \mathrm{C}$. (b) Colony diameter of the WT, $\triangle$ FocM35_1 and $\Delta$ FocM35_1-C strains shown in a. (c) Disease phenotype and (d) disease index distribution in banana plantlets inoculated with the WT, $\triangle$ FocM35_1 and $\Delta$ FocM35_1-C strains at 30 days post inoculation (dpi). (e) Fungal growth in banana roots, determined by quantitative polymerase chain reaction (qPCR). Data presented in (b) and (e) are means \pm SDs from three independent experiments. The letters above the bars indicate statistically significant differences at $p<0.05$ (Student's $t$-test).

It has been reported that tolerance against oxidative stress and osmotic stress is associated with the virulence of pathogenic fungi [23,24]. We, therefore, measured the sensitivity of $\triangle$ FocM35_1 and WT strains to oxidative stress on minimal medium (MM) plates supplemented with $20 \mathrm{mM} \mathrm{H}_{2} \mathrm{O}_{2}$ and to osmotic stress on MM plates supplemented with $1.5 \mathrm{M} \mathrm{NaCl}$. The deletion mutant $\Delta$ FocM35_1 exhibited a significant decrease of tolerances to both oxidative stress mediated by $\mathrm{H}_{2} \mathrm{O}_{2}$ and osmotic stress mediated by $\mathrm{NaCl}$, compared to that of the WT (Figure S4c,d). Taken together, these results indicated that the $\Delta$ FocM35 mutant strain was sensitive to both oxidative and osmotic stress, which may have contributed to its loss of virulence.

\subsection{FocM35_1 Could Decrease Banana Chitinase Activity and Target at MaChiA}

It was reported that M36 metalloprotease was able to target and cleave class IV plant chitinase $[15,16]$. We investigated whether FocM35_1 could influence banana chitinase activity. FocM35_1 protein was expressed in E. coli and purified (Figure S5). Then the recombinant His-FocM35_1 was applied to banana embryogenic cell suspensions (ECSs). Treatment with His-FocM35_1 protein significantly decreased banana chitinase activity dose-dependently (Figure 4a). As a control, chitinase activity of ECSs incubated with BSA or elution buffer remained unchanged (Figure 4a). 
(a)

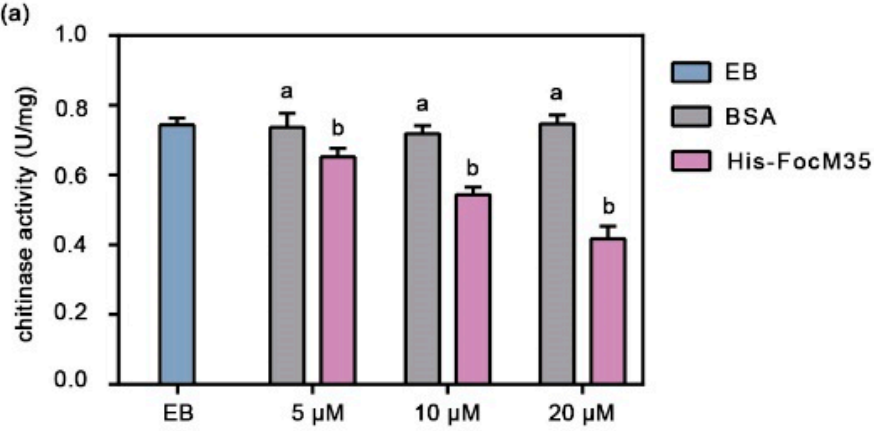

(b)

mVenus

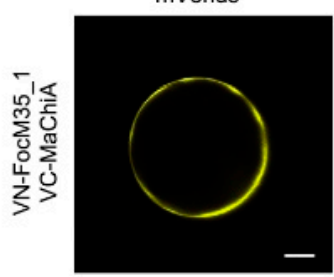

ECFP

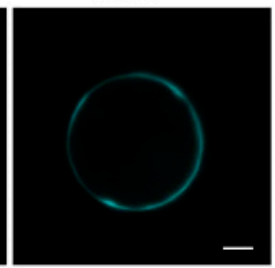

Bright

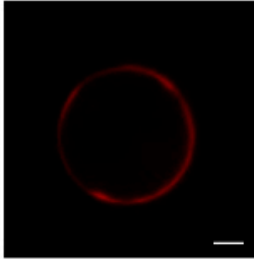

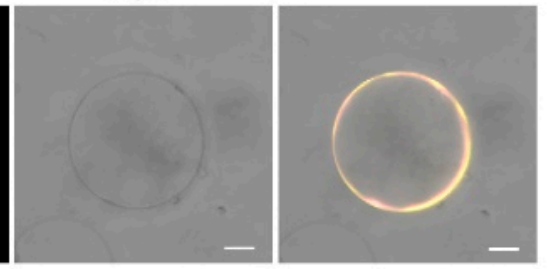

Figure 4. Metalloprotease activity of FocM35_1. (a) Treatment with His-FocM35_1 protein decreased chitinase activity. Banana embryonic suspension cells were incubated with elusion buffer (EB), different concentrations of BSA or HisFocM35_1 protein individually. Chitinase activity was assayed. All data are means \pm SDs from three independent experiments. Different letters above the histograms indicate statistically significant differences at $p<0.05$ (Student's t-test). (b) FocM35_1 and MaChiA interaction detected in rice membrane by bimolecular fluorescence complementation (BiFC) assay. The mVenus channel exhibit Venus fluorescence reflecting the direct interaction of FocM35_1 and MaChiA. Bars $=10 \mu \mathrm{m}$.

To test whether FocM35_1 directly interacts with chitinase, we first analyzed the expression patterns of genes encoding banana chitinases with GH19 (glycosyl hydrolase family 19) domain during the infection of Cavendish banana by Foc race 1 and Foc TR4, respectively (data not published). Based on the expression patterns obtained from the RNA-Seq data, these genes were classified into two large clusters (Figure S6). Four genes in cluster II (Ma05_17850, Ma03_28030, Ma06_31980, and Ma09_20710) are highly expressed during the pathogen infection (Figure S6). Since Ma09_20710 showed higher homology with ZmChiA [15] than the other members in this chitinase family, it was designated as MaChiA and used to investigate its interaction with FocM35_1. Biomolecular fluorescence complementation (BiFC) assay was performed in rice protoplasts to test the interaction between FocM35_1 and MaChiA. FocM35_1-nsp (with no signal peptide) and MaChiA fragments were fused with the split $\mathrm{N}$-terminal and $\mathrm{C}$-terminal in the vectors of pRTVnVN and pRTVnVC, respectively. After co-transfection, the ECFP and mCherry fluorescence indicated successful transfection and expression of the two plasmids. Then we detected clear yellow fluorescence signals on the plasma membrane of the transfected protoplasts (Figure $4 \mathrm{~b}$ ), indicating the formation of reconstituted mVenus through the FocM35_1-MaChiA interaction. Therefore, FocM35_1 seems able to bind the banana chitinase MaChiA directly.

\subsection{The Metalloprotease Activity of FocM35_1 Is Required for Triggering Cell Death in N. benthamiana}

To investigate the influence of FocM35_1 on plant immunity, we performed Agrobacterium tumefaciens-mediated transient expression of FocM35_1 in N. benthamiana leaves to test whether they trigger cell death. Empty vector and the oomycete PAMP (pathogenassociated molecular pattern) elicitor INF1 were also expressed as controls (Figure 5a). We found that FocM35_1 expression induced cell death in N. benthamiana leaves as well as the INF1 control, but not the empty vector control (Figure 5b). Next, we tested whether the signal peptide and the predicted catalytic site (amino acid position 303) of FocM35_1 are responsible for causing cell death in N. benthamiana. A signal peptide (1-15 bp)-deleted 
mutant (FocM35_1-nsp) and a catalytic site missense (303rd amino acid to alanine) mutant were constructed (Figure 5a) and transiently expressed in $N$. benthamiana. We found that FocM35_1-nsp and FocM35_1 ${ }^{\text {E303A }}$ failed to induce cell death (Figure 5b). Immunoblotting analysis confirmed that all the corresponding proteins were successfully expressed in $N$. benthamiana leaves (Figure 5c), except for FocM35_1-nsp. Cell death induction activities were determined by ion leakage measurement and were consistent with the cell death phenotypes (Figure 5d). Furthermore, it was found that FocM35_1 aggregated at the apoplastic space of $N$. benthamiana cells through subcellular localization studies (Figure 5e). These results suggested that FocM35_1 is secreted into the apoplast and triggers cell death in the host plant. In addition, the signal peptide, and the enzymatic activity of FocM35_1 are required for its cell death-promoting function.
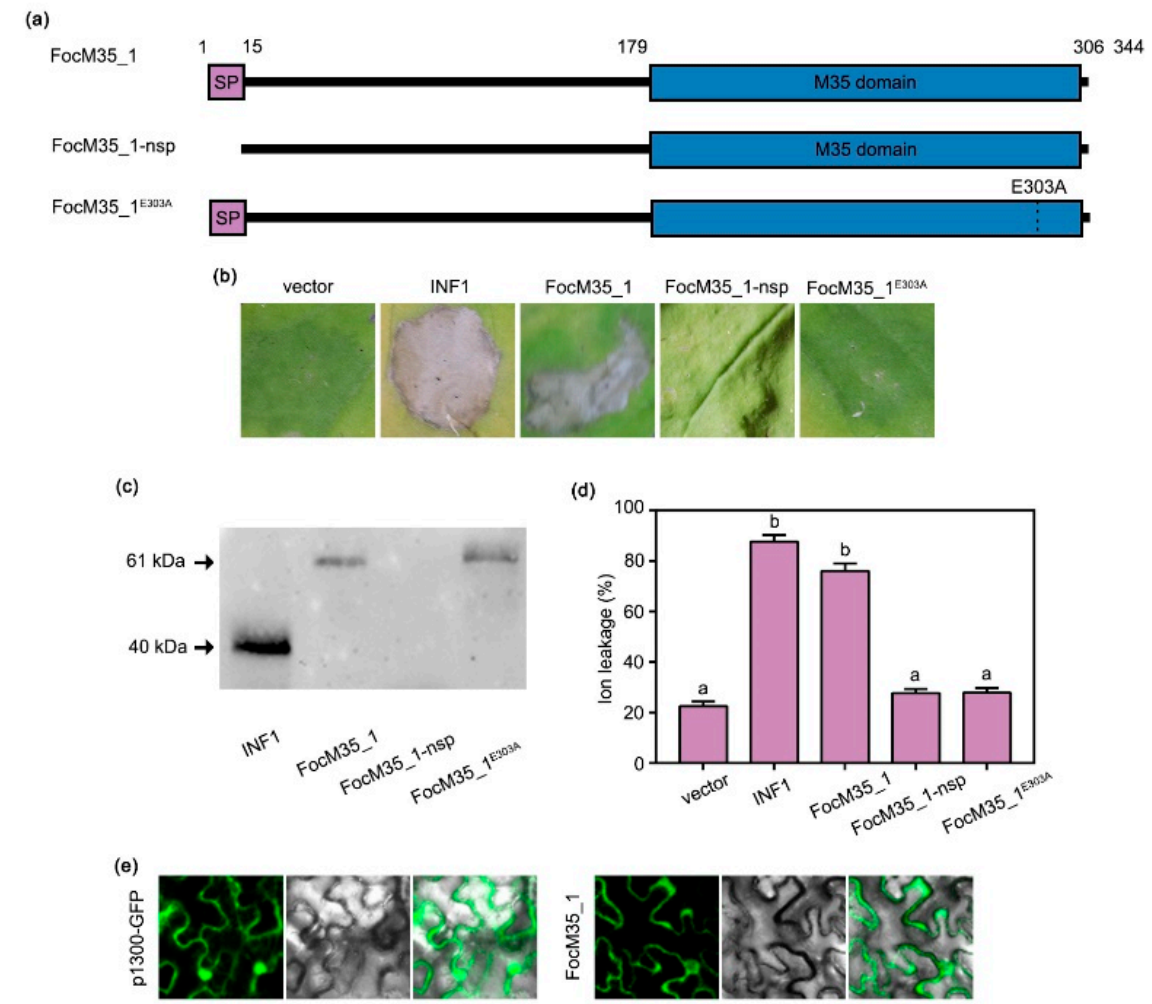

Figure 5. The signal peptide and enzymatic activity of FocM35_1 is required for its cell deathpromoting effects. (a) Schematic illustration of FocM35_1 deletion and point mutation mutants used in this study. (b) Nicotiana benthamiana leaves were infiltrated with Agrobacterium tumefaciens carrying vector, INF1, FocM35_1 and its mutants. Photographs were taken at 5 dpi. Similar results were obtained in two additional experiments. (c) Western blotting detection of control, INF1, FocM35_1 and its mutants using anti-GFP. (d) Quantification of cell death by electrolyte leakage measurement. Different letters above the histograms indicate statistically significant differences at $p<0.05$ (Student's $t$-test). (e) Subcellular localization of FocM35_1 by transient expression of green fluorescent protein (GFP)-fused FocM35_1 in N. benthamiana leaves. Photographs were taken at 2 dpi. The vector was used as control.

\subsection{FocM35_1 Suppresses INF1-Induced Cell Death in N. benthamiana and Accelerates Infection Process of Foc TR4}

We next investigated whether FocM35_1 can suppress plant immune responses. Since the oomycete PAMP elicitor INF1 strongly induces cell death in N. benthamiana [25], we examined the possible effect of FocM35_1 on the INF1-elicited immune response in $N$. benthamiana. Transient expression of INF1 by agroinfiltration induced rapid HR cell death in N. benthamiana within $3 \mathrm{~d}$ post-inoculation (dpi), which is faster than FocM35_1induced cell death that appeared at 5 dpi (Figure 6a). Furthermore, it was found that when 
N. benthamiana leaves were pre-infiltrated with Agrobacterium harboring a plasmid expressing FocM35_1, the INF1-induced cell death in the same leaf was significantly delayed from 3 dpi to 6 dpi (Figure 6a). Moreover, the mutant FocM35_1 ${ }^{\mathrm{E} 303 \mathrm{~A}}$ failed to suppress the INF1-induced cell death, indicating the enzymatic activity of FocM35_1 is required for the suppression of INF1-induced cell death (Figure 6a). Western blot analysis showed that INF1 was co-expressed with GFP, FocM35_1, and FocM35_1 ${ }^{\mathrm{E} 303 \mathrm{~A}}$ in N. benthamiana leaves (Figure $6 \mathrm{~b}$ ). Electrolyte leakage in $N$. benthamiana triggered by INF1 was significantly attenuated in the presence of FocM35_1 (Figure 6c). Collectively, these data suggest that FocM35_1 suppresses INF1-induced cell death in a plant.

(a)

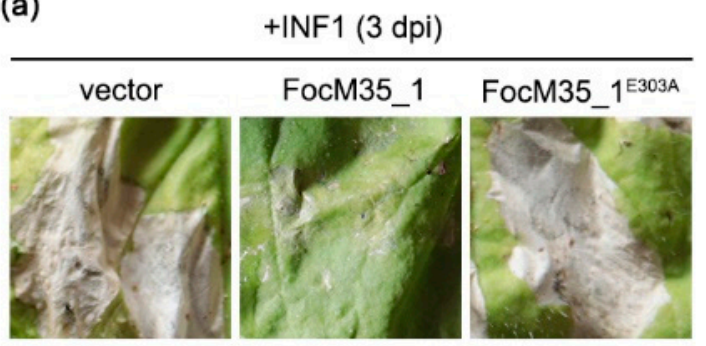

(b)

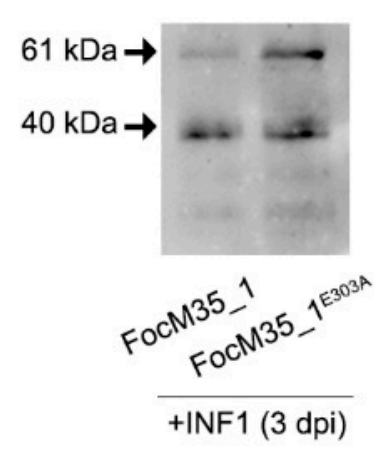

(c) 100

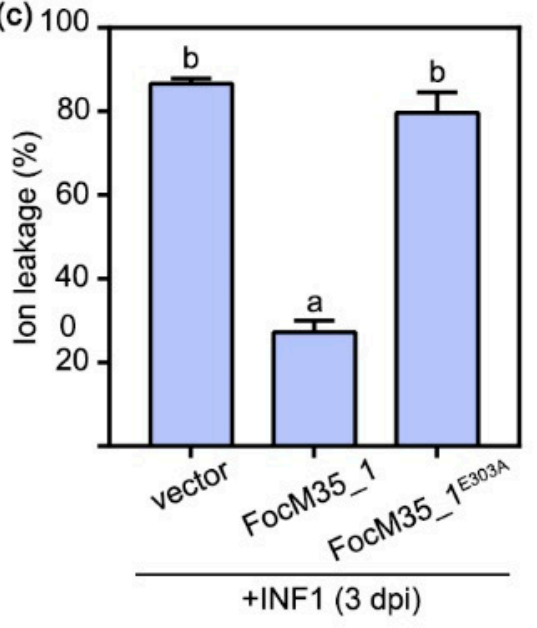

Figure 6. FocM35_1 suppress INF1-induced cell death and its enzymatic activity is required for immunosuppressive ability. (a) 1 day after infiltration with A. tumefaciens carrying vector, FocM35_1 and FocM35_1-nsp (without signal peptide), N. benthamiana leaves were further challenged with A. tumefaciens carrying INF1 at the same sites. The photographs were taken at 3 dpi. Similar results were obtained in two additional experiments. (b) Western blotting detection of FocM35_1 and INF1 using anti-GFP. (c) Quantification of cell death by electrolyte leakage measurement. Different letters above the histograms indicate statistically significant differences at $p<0.05$ (Student's $t$-test).

To further examine the biological function of FocM35_1, banana leaves were inoculated with the recombinant FocM35_1 protein before the inoculation of Foc TR4. It was found the lesion formation in banana leaves was greatly enhanced by this co-inoculation of recombinant FocM35_1 and Foc TR4 (Figure 7). These findings suggest that FocM35_1 enhances the Foc TR4 infection by suppressing the plant immune response to promote the lesion formation, and the suppression is likely targeting the INF1-induced HR response. 


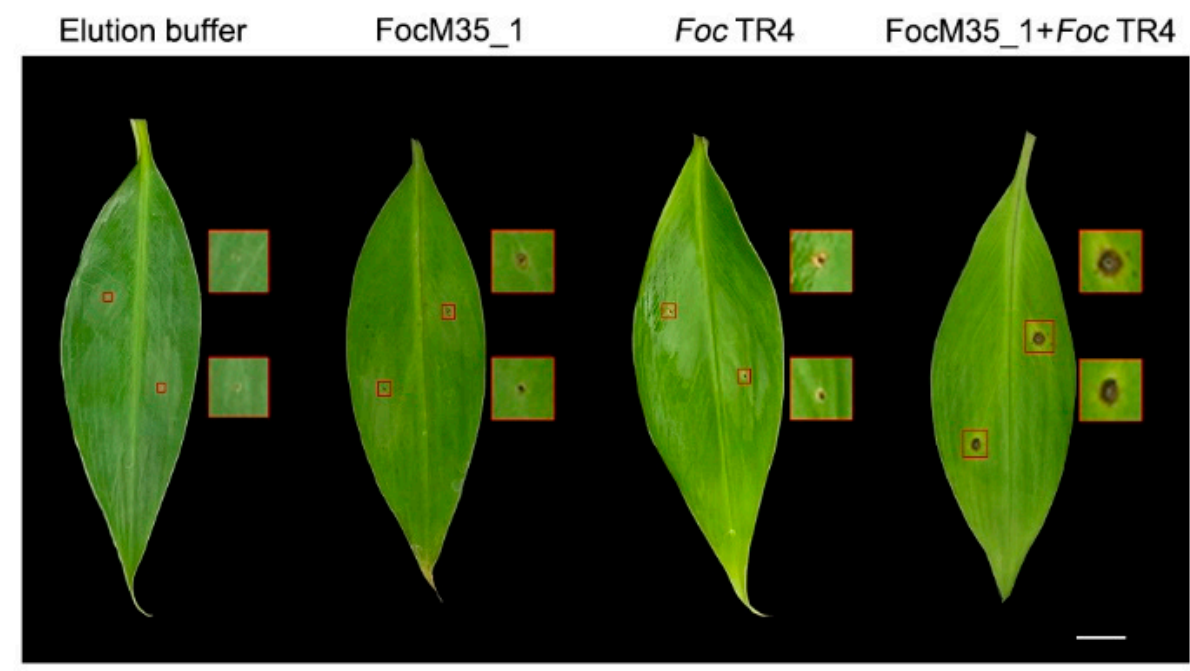

Figure 7. FocM35_1 promoted lesion formation of Foc TR4 in banana leaves. Banana leaves were infiltrated with elution buffer, recombinant FocM35_1, Foc TR4 or recombinant FocM35_1 together with Foc TR4. Photographs were taken 4 dpi. The areas within the red squares are enlarged into pictures at the right side. $B a r=1 \mathrm{~cm}$.

\section{Discussion}

To date, few virulence effector proteins have been identified to be essential for Foc TR4 virulence. Taking advantage of the well-annotated genomic sequence of Foc TR4, hundreds of effector-coding genes were predicted (data not published). Further analysis of the transcriptome of Foc TR4 infection processes revealed many effector candidates whose expressions are highly induced during different infection stages (data not published). One of the putative effector proteins, FocM35_1, was predicted to function as a metalloprotease as it contains the conserved HEXXH motif. Expression pattern analysis based on qRT-PCR and previous transcriptome profiling showed that FocM35_1 was highly expressed during the early stage of infection. The knockout of FocM35_1 significantly reduced Foc TR4 virulence in banana plantlets. In this study, we verified that FocM35_1 contains a predicted signal peptide, and its secretion was verified using a yeast secretion system. In addition, subcellular localization assays showed that FocM35_1 was mainly accumulated in the apoplast, indicating that FocM35_1 is secreted into the apoplast region by Foc TR4 and plays a role in pathogenesis.

Metalloproteases have been implicated as important virulence factors in both bacterial and fungal pathogens $[15,26,27]$. Previous studies described that an M36 metalloprotease FvFly1 of F. verticillioides cleaves $\mathrm{ZmChiA}$ at the hinge domain to release the chitin-binding domain and the hydrolase domain as byproducts [14]. Another M36 metalloprotease, FoMep1 of Fol, was also demonstrated to target the same hinge domain site of tomato SlChi1 [17]. In addition, FoMep1 showed a synergized function with the serine protease FoSep1 to influence the pathogenesis of Fol [17]. Although how M36 metalloproteases modulate the host IV class chitinases activity during pathogenic development was wellstudied, it is still unknown how M35 metalloproteases of phytopathogens interact with host targets. M35 metalloproteases share the same HEXXH enzyme domain with M36 metalloproteases [19] and may also target chitinase under a similar mechanism. Therefore, this study tested and showed that FocM35_1 was able to target banana chitinase MaChiA and decrease chitinase activity, and deletion of FocM35_1 reduced virulence of Foc TR4, indicating the importance of FocM35_1 in pathogenesis through its interaction with plant chitinase. Whether FocM35_1 could cleave MaChiA remains to be further studied in the future. We also found another M35 metalloprotease (FocM35_2) in the Foc genome, but whether FocM35_2 is also involved in fungal virulence should be determined in the future.

At the same time, we performed a range of agroinfiltration assays and found FocM35_1 was able to trigger cell death in N. benthamiana leaves. We also found the ability of 
FocM35_1 to trigger cell death depends on its signal peptide and metalloprotease activity. We propose that FocM35_1 is secreted into extracellular spaces, targeting components of plant immunity that further trigger downstream cell death. Cell death is considered a defense response, as it can prevent pathogens' further invasion by eliciting host immune response [28]. However, in hemi-trophic and necrotrophic pathogens, cell death induced by some effectors and toxins is also regarded as a strategy to facilitate the invasion of pathogens [29]. Phytophthora parasitica is a hemi-trophic phytopathogen, and its cysteine proteases PpCys44 and PpCys45 positively promote pathogen virulence during infection by triggering plant cell death [30]. Here, we reported FocM35_1 induced cell death in $N$. benthamiana leaves and suppressed the INF1-induced cell death. INF1 is a well-known oomycete PAMP elicitor, which strongly induces cell death in N. benthamiana and triggers plant immune defenses [25]. One possible explanation for how FocM35_1 suppressed INF1-induced cell death while still induced cell death by itself in N. benthamiana is that FocM35_1 may target and cleave a receptor of INF1, blocking the INF1-induced cell death, while FocM35_1-triggered cell death may be deployed by a pathogen to enhance virulence and promote fungal invasion. A similar mechanism was reported on the effector CoNIS1 of Colletotrichum orbiculare [31]. CoNIS1 interact with PRR-associated kinases BAK1 and BIK1, which are the receptors of INF1, thereby inhibiting INF1-induced cell death [31]. To better elucidate the underlying mechanism of FocM35_1 in suppression of the INF1-associated hypersensitive response, future studies should be performed to investigate the interaction between FocM35_1 and INF1-related plant defense pathway.

Interestingly, pretreatments of recombinant FocM35_1 promoted the invasion of Foc TR4, indicating FocM35_1 induces cell death to further enhance fungal virulence. Since Foc TR4 is a typical hemi-biotrophic plant pathogen, cell death may facilitate pathogen infection. It implied that FocM35_1 may play an important role at an early stage of infection by promoting the transition from biotrophy to necrotrophy lifestyle. Overall, the results of this study not only revealed the pathogenesis mechanism of a new virulence effector of Foc TR4 but also demonstrated the significant role of M35 family metalloprotease in fungal virulence.

\section{Materials and Methods}

\subsection{Plant Growth Conditions, Fungal Strains and Growth Conditions}

'Cavendish' banana (AAA) cv 'Brazilian' plantlets with 5 to 6 leaves $(c .20 \mathrm{~cm}$ height) were grown in a temperature-controlled glasshouse (14 h light and $10 \mathrm{~h}$ dark cycle, at $28{ }^{\circ} \mathrm{C}$ with $40 \%$ humidity). N. benthamiana was grown in the growth chamber at $25^{\circ} \mathrm{C}$. The wild-type (WT) and mutants of Fusarium oxysporum f. sp. cubense TR4 strain II5 were cultured on potato dextrose agar (PDA) or in potato dextrose broth (PDB) at $28^{\circ} \mathrm{C}$.

\subsection{Bioinformatics Analysis}

Gene ID of FocM35_1 in NCBI is 42027094. A search of M35 metalloprotease proteins in 12 Fusarium species was performed using a BlastP search on the NCBI non-redundant (nr) database. ClustalX 2.1 [32] was used for protein alignment and, ESPript 3.0 [33] was used for drawing alignment graphs with secondary structure information. A phylogenetic tree was constructed using MEGA X [34] maximum likelihood method with 1000 bootstrap replications. SignalP v. 4.0 [35] was used for signal peptide prediction. The expression patterns of banana chitinases were based on the RNA-seq data for Cavendish banana infected with Foc race 1 and Foc TR4, respectively, at 18, 32, 56 h post-inoculation (hpi) (data not published). The heatmap of all chitinase-coding genes was generated with Tbtools [36] using fragments per kilobase of transcript per million mapped reads (FPKM) values of each gene.

\subsection{Signal Peptide Secretion Test}

The function of predicted signal peptides of FocM35_1 was evaluated according to a previously published protocol [37]. Briefly, the predicted signal peptide of FocM35_1 was fused into pSUC2 vector and, then the constructs were transformed into yeast Saccharomyces 
cerevisiae YTK12 strain. All transformants were cultured on CMD-W medium (6.7 g/L yeast nitrogen base without amino acids, $0.74 \mathrm{~g} / \mathrm{L}$-Trp DO supplement, $20 \mathrm{~g} / \mathrm{L}$ sucrose, $1 \mathrm{~g} / \mathrm{L}$ glucose, and $20 \mathrm{~g} / \mathrm{L}$ Agar A) to select positive colonies. Colorimetric change (to red) after adding $0.1 \%$ TCC solution is observed if the signal peptide is functional.

\subsection{Construction of Gene Replacement and Complementary Strain}

The $\Delta$ FocM35_1 mutant strain was constructed by homologous recombination as previously described [12]. Briefly, the $5^{\prime}$ - and $3^{\prime}$-flanking sequences of the FocM35_1 gene were amplified using the genomic DNA of II5 as a template with the Phanta Max polymerase (Vazyme, China). The primers used in this study were listed in Table S1. The FocM35_1 gene was replaced by a hygromycin-resistance cassette $(\mathrm{HPH})$, which was driven by a constitutive TrpC promoter amplified from the $\mathrm{pCT74}$ vector. PCR products were transformed into protoplasts of WT strain II5 by protoplast transformation mediated by polyethylene glycol (PEG). The putative deletion mutants were identified by PCR analysis and further confirmed by Southern blot. Gene complementation vector was constructed by introducing native promoter-FocM35_1 fragment into pYF11 (Geneticin resistance) vector, and the complemented strain was also verified by PCR amplification.

\subsection{Phenotyping, Stress Sensitivity and Cellophane Membrane Assays}

In order to determine whether there is any phenotypic difference between WT, $\Delta$ FocM35_1, and $\Delta$ FocM35-C, $5 \mathrm{~mm}$ mycelial plug of each strain was inoculated on PDA plate for five days in the incubator at $28{ }^{\circ} \mathrm{C}$. To determine the difference in stress responses between WT and mutants, strains were cultured on PDA plates supplemented with the final concentrations of $1.5 \mathrm{M} \mathrm{NaCl}$ or $20 \mathrm{mM} \mathrm{H}_{2} \mathrm{O}_{2}$ for five days at $28^{\circ} \mathrm{C}$. In addition, the penetration ability of each strain was tested on the cellophane membrane as described previously [12]. The experiments were repeated three times.

\subsection{Pathogenicity Assays and Fungal Biomass Estimation}

'Cavendish' banana (AAA) cv 'Brazilian' plantlets with 5 to 6 leaves $(c .20 \mathrm{~cm}$ height) were inoculated with Foc strains conidial suspensions at a concentration of $1 \times 10^{7}$ conidia $\mathrm{L}^{-1}$, with clear water as the negative control. In order to evaluate the pathogenicity, the disease severity of each experimental plant was evaluated as previously reported [38].

Quantitative PCR (qPCR) was performed to determine the fungal biomass in the rhizome [38]. Elongation factor 1- $\alpha$ (FocEF1 $\alpha$ ) gene of Foc TR4 and banana actin (MusaActin) gene were used to quantify fungi biomass and plant, respectively. (Table S1)

\subsection{RNA Extraction and Quantitative RT-PCR Analysis}

Total RNA of infected banana roots was extracted using a SteadyPure Plant RNA Extraction Kit (Accurate Biology, Hunan, China) following the manufacturer's instructions. Genomic DNA was eliminated, and reverse transcription reaction was carried out using an Evo M-MLV RT Kit (Accurate Biology, Hunan, China). To analyze the expression pattern of FocM35_1 during infection, qRT-PCR analysis was performed in Step Two real-time PCR system (Accurate Biology, Hunan, China) with the SYBR ${ }^{\circledR}$ Green Premix (Applied Accurate Biology). FocEF1 $\alpha$ was used as an internal control to normalize the data and, the relative expressions of genes were calculated using the $2^{-\Delta \Delta C T}$ method. The gene-specific primers used for qRT-PCR are listed in Table S1.

\subsection{Scanning Electron Microscopy Observation}

For SEM observation, 'Cavendish' banana (AAA) cv 'Brazilian' plantlets with 5 to 6 leaves (c. $20 \mathrm{~cm}$ height) were inoculated with Foc strains conidial suspensions at a concentration of $1 \times 10^{7}$ conidia/L. The root samples were collected $48 \mathrm{~h}$ later and observed using a Hitachi Model S-3400N scanning electron microscope (Hitachi, Tokyo, Japan). 


\subsection{Protein Expression}

The cDNA of FocM35_1 was amplified and cloned into the expression vector pET28a-His for expression in E. coli Rosetta (DE3). Transformed cells were cultured overnight at $37^{\circ} \mathrm{C}$ in LB medium and induced with isopropyl $\beta$-D-1-thiogalactopyranoside (IPTG) for $24 \mathrm{~h}$ at $28{ }^{\circ} \mathrm{C}$. The cells were lysed by supersonic treatment, and the supernatant from the lysate was applied to a Ni-NTA column (Transgene Biotech, Beijing, China). Protein concentration was determined using the microtiter plate method (Sangon Biotech, Shanghai, China) and was also confirmed by SDS-PAGE gel stained with Coomassie Blue.

\subsection{Chitinase Activity Assays}

The effect of FocM35_1 on banana chitinase was determined on banana ECSs. ECSs were incubated with purified His-FocM35_1 protein of different concentrations $(5,10,20 \mu \mathrm{M})$ at $28^{\circ} \mathrm{C}$ for $1 \mathrm{~h}$. The control treatment included equal concentrations of BSA or elusion buffer alone. The protein isolation of banana ECSs was performed as previously described [38].

Chitinase activity was determined using $40 \mathrm{mM}$ 4-Nitrophenyl $\beta$-D- $N, N^{\prime}, N^{\prime \prime}$ triacetylchitotriose (Sigma, St. Louis, MO, USA) as a substrate. $10 \mathrm{~mL}$ ECSs extract at $0.2 \mathrm{mg} / \mathrm{mL}$ was mixed with an equal volume of substrate solution and incubated at $37^{\circ} \mathrm{C}$ for $1 \mathrm{~h}$. Then the reaction was quenched by the addition of $20 \mathrm{~mL}$ of $1 \mathrm{M} \mathrm{Gly-NaOH}$ ( $\mathrm{pH}$ 10.2). The release of the chromophore $\rho$-nitrophenol (pNP) was measured at $405 \mathrm{~nm}$. One unit $(\mathrm{U})$ of activity per $\mathrm{mg}$ chitinase $(\mathrm{U} / \mathrm{mg})$ was defined as the release of $1 \mathrm{mmol}$ of $\mathrm{pNP} / \mathrm{mg}$ per minute under the assay conditions.

\subsection{Rice Protoplasts Isolation and Transfection for BiFC (Bimolecular Fluorescence Complementation) Assay}

The interaction between FocM35_1 with MaChiA was verified using the BiFC system. FocM35_1 and MaChiA gene was introduced into pRTVnVN and pRTVnVC vectors, respectively. The isolation, transfection, and fluorescence detection were carried out according to the method described before [39].

\subsection{Subcellular Localization Assay}

To determine the subcellular localization of FocM35_1, A. tumefaciens EHA105 containing the vector pCAMBIA1300 with FocM35_1 was transiently expressed in N. benthamiana in an infiltration medium with an $\mathrm{OD}_{600}$ of 1.0. EHA105 carrying the empty pCAMBIA1300 vector was used as control.

At two days post-infiltration, epidermal tissues of tobacco leaves were sampled and observed with a laser confocal microscope (LSM 710, Carl Zeiss, Oberkochen, Germany).

\subsection{Agroinfiltration Assays}

The constructed vectors pCAMBIA1300 carrying FocM35_1 (with or without signal peptide) or, FocM35_1 ${ }^{\mathrm{E} 303 \mathrm{~A}}$ (catalytic site mutation) were transformed into A. tumefaciens EHA105, respectively. Overnight-cultured recombinant strains of $A$. tumefaciens cells were infiltrated into the leaves of 6-week-old N. benthamiana plants, and the same Agrobacterium strain harboring the empty pCAMBIA1300 vector was used as a negative control, with INF1 as a positive control.

In order to test whether FocM35_1 could suppress INF1-induced cell death, at one day after infiltration with $A$. tumefaciens carrying FocM35_1, the infiltration site was further infiltrated with a recombinant $A$. tumefaciens carrying FocM35_1 or FocM35_1 ${ }^{\mathrm{E} 303 \mathrm{~A}}$. All tests were performed three times.

The total proteins of $N$. benthamiana leaves were extracted with a plant protein extraction kit (Sangon Biotech, Shanghai, China) according to the manufacturer's instruction. Then the proteins were analyzed by SDS-PAGE and immunoblotting. 


\subsection{Banana Leaf Infection}

Leaves of 'Cavendish' banana (AAA) cv 'Brazilian' plantlets were treated with recombinant FocM35_1 or elution buffer (phosphate buffer saline, pH 7.0). One hour after treatment, Foc spore suspension was infiltrated into the same area of leaves. Disease phenotype was observed at $5 \mathrm{~d}$ post-inoculation. The experiments were repeated three times.

Supplementary Materials: Supplementary materials can be found at https:/ /www.mdpi.com/ article/10.3390/pathogens10060670/s1. Table S1: Primers used in this study. Figure S1: Multiple sequence alignment of M35 domain and its homologues mainly from Fusarium spp. Figure S2: Functional test of the predicted signal peptide of FocM35_1 using a yeast secretion system. Figure S3: Schematic representation of gene disruption strategy and Southern blotting analyses of the deletion mutant. Figure S4: FocM35_1 is required for successful penetration and colonization and is involved in the response towards oxidative and osmotic stress. Figure S5: Clustering analysis of the expression patterns of banana chitinase genes encoding proteins with GH19 domain, based on RNA-Seq data from banana roots inoculated with Foc TR4. Figure S6: Purification of the recombinant FocM35_1(a) and MaChiA (b).

Author Contributions: Conceptualization, X.Z. and S.L. (Siwen Liu); methodology, C.L. and S.L. (Shuxia Lyu); software, H.H.; validation, B.W.; formal analysis, J.X., W.W., A.V., D.M. and Y.X.; investigation, S.L. (Shuxia Lyu), X.Z., H.H. and B.W.; resources, D.X. and G.F.; data curation, S.L. (Shuxia Lyu); writing—original draft preparation, X.Z.; writing-review and editing, S.L. (Shuxia Lyu) and S.L. (Siwen Liu); supervision, C.L. and S.L. (Siwen Liu); project administration, C.L.; funding acquisition, C.L. All authors have read and agreed to the published version of the manuscript.

Funding: This work was supported by National Key Research and Development Program of China (2018YFD1000300), Guangdong Science and Technology Project (2019B1515120088), Guangdong Provincial Special Fund for Modern Agriculture Industry Technology Innovation Teams (No.2019KJ109), National Banana Industry and Technology System Project (nycytx-33).

Institutional Review Board Statement: Not applicable.

Informed Consent Statement: Not applicable.

Conflicts of Interest: The authors declare no competing interests.

\section{References}

1. Ploetz, R.C. Fusarium wilt of banana. Phytopathology 2015, 105, 1512-1521. [CrossRef] [PubMed]

2. Ploetz, R.C. Panama disease: An old nemesis rears its ugly head part 2: The Cavendish era and beyond. Apsnet Feature Artic. 2005. [CrossRef]

3. García-Bastidas, F.A.; Quintero-Vargas, J.C.; Ayala-Vasquez, M.; Schermer, T.; Seidl, M.F.; Santos-Paiva, M.; Noguera, A.M.; Aguilera-Galvez, C.; Wittenberg, A.; Hofstede, R.; et al. First report of Fusarium wilt tropical race 4 in cavendish bananas caused by Fusarium odoratissimum in Colombia. Plant Dis. 2020, 104, 994. [CrossRef]

4. Molina, A.; Chao, C.-P.; Dusunceli, F.; Beed, F.; Rose, L.J.; Bothma, S.; Pretorius, A.; Vaz, A.; Mondjana, A.; Amugoli, O.M.; et al. Occurrence and spread of the banana fungus Fusarium oxysporum f. sp. cubense TR4 in Mozambique. S. Afr. J. Sci. 2020, 116. [CrossRef]

5. Viljoen, A.; Ma, L.-J.; Molina, A.B. CHAPTER 8: Fusarium Wilt (Panama Disease) and Monoculture in Banana Production: Resurgence of a Century-Old Disease. In Emerging Plant Diseases and Global Food Security; APS press: St. Paul, MN, USA, 2020; pp. 159-184. [CrossRef]

6. Dale, J.; James, A.; Paul, J.Y.; Khanna, H.; Smith, M.; Peraza-Echeverria, S.; Garcia-Bastidas, F.; Kema, G.; Waterhouse, P.; Mengersen, K.; et al. Transgenic Cavendish bananas with resistance to Fusarium wilt tropical race 4. Nat. Commun. $2017,8,1496$. [CrossRef]

7. Dita, M.; Barquero, M.; Heck, D.; Mizubuti, E.S.G.; Staver, C.P. Fusarium wilt of banana: Current knowledge on epidemiology and research needs toward sustainable disease management. Front. Plant Sci. 2018, 9, 1468. [CrossRef]

8. Dangl, J.L.; Horvath, D.M.; Staskawicz, B.J. Pivoting the plant immune system from dissection to deployment. Science 2013, 341, 746-751. [CrossRef]

9. Wang, Y.; Wang, Y. Trick or treat: Microbial pathogens evolved apoplastic effectors modulating plant susceptibility to Infection. Mol. Plant Microbe Interact. 2018, 31, 6-12. [CrossRef]

10. Widinugraheni, S.; Nino-Sanchez, J.; van der Does, H.C.; van Dam, P.; Garcia-Bastidas, F.A.; Subandiyah, S.; Meijer, H.J.G.; Kistler, H.C.; Kema, G.H.J.; Rep, M. A SIX1 homolog in Fusarium oxysporum f.sp. cubense tropical race 4 contributes to virulence towards Cavendish banana. PLoS ONE 2018, 13, e0205896. [CrossRef] 
11. An, B.; Hou, X.; Guo, Y.; Zhao, S.; Luo, H.; He, C.; Wang, Q. The effector SIX8 is required for virulence of Fusarium oxysporum f.sp. cubense tropical race 4 to Cavendish banana. Fungal. Biol. 2019, 123, 423-430. [CrossRef] [PubMed]

12. Liu, S.; Wu, B.; Yang, J.; Bi, F.; Dong, T.; Yang, Q.; Hu, C.; Xiang, D.; Chen, H.; Huang, H.; et al. A cerato-platanin family protein FocCP1 is essential for the penetration and virulence of Fusarium oxysporum f. sp. cubense tropical race 4. Int. J. Mol. Sci. 2019, $20,3785$. [CrossRef] [PubMed]

13. Miyoshi, S.-I.; Shinoda, S. Microbial metalloproteases and pathogenesis. Microbes Infect. 2000, 2, 91-98. [CrossRef]

14. Naumann, T.A.; Wicklow, D.T.; Price, N.P. Identification of a chitinase-modifying protein from Fusarium verticillioides: Truncation of a host resistance protein by a fungalysin metalloprotease. J. Biol. Chem. 2011, 286, 35358-35366. [CrossRef] [PubMed]

15. Okmen, B.; Kemmerich, B.; Hilbig, D.; Wemhoner, R.; Aschenbroich, J.; Perrar, A.; Huesgen, P.F.; Schipper, K.; Doehlemann, G. Dual function of a secreted fungalysin metalloprotease in Ustilago maydis. New Phytol. 2018, 220, 249-261. [CrossRef] [PubMed]

16. Naumann, T.A.; Wicklow, D.T. Chitinase modifying proteins from phylogenetically distinct lineages of Brassica pathogens. Physiol. Mol. Plant Pathol. 2013, 82, 1-9. [CrossRef]

17. Jashni, M.K.; Dols, I.H.; Iida, Y.; Boeren, S.; Beenen, H.G.; Mehrabi, R.; Collemare, J.; de Wit, P.J. Synergistic action of a metalloprotease and a serine protease from Fusarium oxysporum f. sp. lycopersici cleaves chitin-binding tomato chitinases, reduces their antifungal activity, and enhances fungal virulence. Mol. Plant Microbe Interact. 2015, 28, 996-1008. [CrossRef]

18. Takahara, H.; Hacquard, S.; Kombrink, A.; Hughes, H.B.; Halder, V.; Robin, G.P.; Hiruma, K.; Neumann, U.; Shinya, T.; Kombrink, E.; et al. Colletotrichum higginsianum extracellular LysM proteins play dual roles in appressorial function and suppression of chitin-triggered plant immunity. New Phytol. 2016, 211, 1323-1337. [CrossRef]

19. Rawlings, N.D.; Barrett, A.J.; Bateman, A. MEROPS: The peptidase database. Nucleic Acids Res. 2010, 38, D227-D233. [CrossRef]

20. McAuley, K.E.; Jia-Xing, Y.; Dodson, E.J.; Lehmbeck, J.; Ostergaard, P.R.; Wilson, K.S. A quick solution: Ab initio structure determination of a $19 \mathrm{kDa}$ metalloproteinase using ACORN. Acta Cryst. D Biol. Cryst. 2001, 57, 1571-1578. [CrossRef]

21. Dou, D.; Kale, S.D.; Wang, X.; Jiang, R.H.; Bruce, N.A.; Arredondo, F.D.; Zhang, X.; Tyler, B.M. RXLR-mediated entry of Phytophthora sojae effector Avr1b into soybean cells does not require pathogen-encoded machinery. Plant Cell 2008, 20, 1930-1947. [CrossRef]

22. Sarmiento-Villamil, J.L.; Prieto, P.; Klosterman, S.J.; Garcia-Pedrajas, M.D. Characterization of two homeodomain transcription factors with critical but distinct roles in virulence in the vascular pathogen Verticillium dahliae. Mol. Plant Pathol. 2018, 19, 986-1004. [CrossRef] [PubMed]

23. Molina, L.; Kahmann, R. An Ustilago maydis gene involved in $\mathrm{H}_{2} \mathrm{O}_{2}$ detoxification is required for virulence. Plant Cell 2007, 19, 2293-2309. [CrossRef] [PubMed]

24. Zhang, X.W.; Jia, L.J.; Zhang, Y.; Jiang, G.; Li, X.; Zhang, D.; Tang, W.H. In planta stage-specific fungal gene profiling elucidates the molecular strategies of Fusarium graminearum growing inside wheat coleoptiles. Plant Cell 2012, 24, 5159-5176. [CrossRef]

25. Vleeshouwers, V.G.; Driesprong, J.D.; Kamphuis, L.G.; Torto-Alalibo, T.; Van't Slot, K.A.; Govers, F.; Visser, R.G.; Jacobsen, E.; Kamoun, S. Agroinfection-based high-throughput screening reveals specific recognition of INF elicitins in Solanum. Mol. Plant Pathol. 2006, 7, 499-510. [CrossRef] [PubMed]

26. Liehl, P.; Blight, M.; Vodovar, N.; Boccard, F.; Lemaitre, B. Prevalence of local immune response against oral infection in a Drosophila/Pseudomonas infection model. PLoS Pathog. 2006, 2, e56. [CrossRef] [PubMed]

27. Lee, S.A.; Jang, S.H.; Kim, B.H.; Shibata, T.; Yoo, J.; Jung, Y.; Kawabata, S.I.; Lee, B.L. Insecticidal activity of the metalloprotease AprA occurs through suppression of host cellular and humoral immunity. Dev. Comp. Immunol. 2018, 81, 116-126. [CrossRef]

28. Coll, N.S.; Vercammen, D.; Smidler, A.; Clover, C.; Van Breusegem, F.; Dangl, J.L.; Epple, P. Arabidopsis type I metacaspases control cell death. Science 2010, 330, 1393-1397. [CrossRef]

29. Lorang, J.; Kidarsa, T.; Bradford, C.S.; Gilbert, B.; Curtis, M.; Tzeng, S.C.; Maier, C.S.; Wolpert, T.J. Tricking the guard: Exploiting plant defense for disease susceptibility. Science 2012, 338, 659-662. [CrossRef]

30. Zhang, Q.; Li, W.; Yang, J.; Xu, J.; Meng, Y.; Shan, W. Two Phytophthora parasitica cysteine protease genes, PpCys44 and PpCys45, trigger cell death in various Nicotiana spp. and act as virulence factors. Mol. Plant Pathol. 2020, 21, 541-554. [CrossRef]

31. Irieda, H.; Inoue, Y.; Mori, M.; Yamada, K.; Oshikawa, Y.; Saitoh, H.; Uemura, A.; Terauchi, R.; Kitakura, S.; Kosaka, A.; et al. Conserved fungal effector suppresses PAMP-triggered immunity by targeting plant immune kinases. Proc. Natl. Acad. Sci. USA 2019, 116, 496-505. [CrossRef]

32. Larkin, M.A.; Blackshields, G.; Brown, N.P.; Chenna, R.; McGettigan, P.A.; McWilliam, H.; Valentin, F.; Wallace, I.M.; Wilm, A.; Lopez, R.; et al. Clustal W and Clustal X version 2.0. Bioinformatics 2007, 23, 2947-2948. [CrossRef] [PubMed]

33. Robert, X.; Gouet, P. Deciphering key features in protein structures with the new ENDscript server. Nucleic Acids Res. 2014, 42, W320-W324. [CrossRef] [PubMed]

34. Kumar, S.; Stecher, G.; Li, M.; Knyaz, C.; Tamura, K. MEGA X: Molecular evolutionary genetics analysis across computing platforms. Mol. Biol. Evol. 2018, 35, 1547-1549. [CrossRef]

35. Petersen, T.N.; Brunak, S.; von Heijne, G.; Nielsen, H. SignalP 4.0: Discriminating signal peptides from transmembrane regions. Nat. Methods 2011, 8, 785-786. [CrossRef] [PubMed]

36. Chen, C.; Chen, H.; Zhang, Y.; Thomas, H.R.; Frank, M.H.; He, Y.; Xia, R. TBtools: An integrative toolkit developed for interactive analyses of big biological data. Mol. Plant 2020, 13, 1194-1202. [CrossRef]

37. Yin, W.; Wang, Y.; Chen, T.; Lin, Y.; Luo, C. Functional Evaluation of the Signal Peptides of Secreted Proteins. Bio-Protoc. 2018, 8. [CrossRef] 
38. Liu, S.; Li, J.; Zhang, Y.; Liu, N.; Viljoen, A.; Mostert, D.; Zuo, C.; Hu, C.; Bi, F.; Gao, H.; et al. Fusaric acid instigates the invasion of banana by Fusarium oxysporum f. sp. cubense TR4. New Phytol. 2020, 225, 913-929. [CrossRef] [PubMed]

39. He, F.; Zhang, F.; Sun, W.; Ning, Y.; Wang, G.L. A versatile vector toolkit for functional analysis of rice genes. Rice 2018, 11, 27. [CrossRef] 